\title{
Visualização interativa de modelos BIM em tablets: comparação de aplicativos de uso livre
}

\author{
Interactive visualization of BIM models at tablets. Free apps comparing \\ - Diego Leite da Silva \\ UFPel, Brasil \\ diegols@gmail.com \\ - Felipe Etchegaray Heidrich \\ UFPel, Brasil, \\ felipeheidrich@gmail.com
}

\begin{abstract}
This investigation aims to contribute in the analysis and visualization three-dimensional digital models in the exercises of the disciplines of architectural project by identifying new possibilities to interact with these models using tablets and a free application that allows viewing of file standard of digital models usually developed at BIM software.
\end{abstract}

Keywords: BIM, Visualização Interativa, Aplicativo de uso livre

\section{Introdução}

A mudança de metodologia de desenho arquitetônico baseada em CAD para uma baseada em BIM alterou um sistema que simulava o desenho a mão para outro que simula o processo de construção. O primeiro, fazia uso de elementos gráficos bidimensionais auxiliados por computador, já o segundo, utiliza-se de elementos gráficos tridimensionais com diferentes tipos de informações construtivas. Assim, as informações gráficas deixaram de ser um conjunto de linhas para serem modelos tridimensionais de elementos construtivos. (Grabowski, 2000)

Essa tridimensionalidade é representada por um sistema BIM, que mantém um banco de dados onde exibe a geometria dos elementos construtivos em três dimensões e armazena seus atributos, transmitindo mais informações que os modelos CAD tradicionais. Como os elementos são paramétricos, é possível alterá-los e obter atualizações em tempo real em todo o projeto. Esse processo estimula a experimentação, diminui conflitos entre elementos construtivos, facilita revisões e aumenta a produtividade (Florio, 2007).

Desta forma, os modelos BIM como uma simulação digital tridimensional da edificação a ser construída podem ter enfoques distintos quanto à complexidade ou nível de detalhes das informações que contém, entretanto, quando este modelo passa a ser usado para extração de desenhos bidimensionais em papel, muitas das suas possibilidades de visualização são desconsideradas.

Neste sentido, como observa Levy (1996, p. 41): "Considerar o computador apenas como um instrumento a mais para produzir textos, sons ou imagens sobre suporte fixo equivale a negar sua fecundidade propriamente cultural, ou seja, o aparecimento de novos gêneros ligados à interatividade".

Segundo o mesmo autor "o termo "interatividade" em geral ressalta a participação ativa do beneficiário de uma transação de informação" (LEVY, 1999, p. 79), o que não aconteceria com informações impressas em papel necessitando-se de outro meio de apresentação desta informação.

Além disto, a visualização pode ajudar na aquisição de informação, ampliando a capacidade de compreender, ideia que, segundo Pimentel et al (2013), é expressa por diferentes teóricos contemporâneos. Além disto, conforme comenta Checcucci et al (2013) estudos demonstram uma crescente introdução do paradigma BIM nos cursos de graduação em arquitetura e em engenharia no Brasil.

Devido a isto, o presente estudo considera importante que a utilização dos modelos BIM ultrapasse a geração de desenhos bidimensionais ou tabelas através de uma visualização interativa deste modelo tridimensional, apoiando-se no uso de tablets e em aplicativos específicos para este tipo de modelo.

Sendo assim, a pergunta que gerou o presente estudo foi: Que visualização e interatividade com os modelos BIM são possíveis através da adoção de aplicativos de uso livre? Para tanto, o estudo realizado teve como objetivo principal: verificar as possibilidades de visualização e interatividade com os modelos BIM através da comparação de aplicativos uso livre para tablets. 


\section{Metodologia}

A metodologia escolhida para a comparação entre os aplicativos tomou como base o estudo realizado por Catani et al (2007). Este estudo tinha como problema de pesquisa: identificar qual ferramenta do software AutoCAD era mais viável para modelagem tridimensional: o Arqui_3D ou AutoCAD 3D.

Para isto o estudo de Catani et al (2007), através da geração de modelos tridimensionais de duas edificações com o uso do Arqui_3D e do AutoCAD 3D, comparou os resultados obtidos, levando em conta os seguintes critérios: velocidade de representação dos modelos tridimensionais; dificuldade de manipulação dos modelos representados; e tamanho final do arquivo. Esta metodologia e os três critérios foram então adequados ao tipo de ferramentas que o presente estudo tinha como objetivo comparar.

Quanto ao item referente ao arquivo gerado, a quantidade de informação foi adotada como variável de comparação pela metodologia utilizada como referência, mas optou-se por substituí-lo no presente estudo para os tipos de arquivos passíveis de visualização nos aplicativos analisados.

O item referente à dificuldade de manipulação dos modelos representados foi adequado para usabilidade das ferramentas do aplicativo, levando em consideração a dificuldade ou facilidade de manipulação das ferramentas presentes nos aplicativos comparados.

Para o terceiro critério, velocidade de desenvolvimento, que dizia respeito à principal função dos softwares comparados por Catani et al. (2007), ou seja, desenvolvimento da modelagem tridimensional, o presente estudo adequou o critério também para principal função dos aplicativos comparados (possibilidades de visualização e interação).

Para a escolha dos aplicativos a serem comparados, inicialmente fez-se um levantamento que visava apontar onde estes aplicativos relacionados poderiam ser encontrados, em quais 'lojas' de aplicativos e para quais dispositivos (smartphones, tablets). Isso porque, dependendo do sistema operacional, bem como da sua versão, o aplicativo pode ou não ser disponibilizado.

Nesta pesquisa constatou-se que a loja de aplicativos para o sistema operacional Android, a Google Play, disponibiliza (quando da realização do estudo) apenas o aplicativo BIMx, da Graphisoft, inviabilizando a realização de comparação com outros aplicativos com as características definidas pelo estudo. Devido a isto, neste estudo, foi desconsiderada a possibilidade de investigar os aplicativos para o referido sistema operacional.

No levantamento de aplicativos para o sistema operacional iOS (versões 6, 7 e 8), realizado no site da loja da Apple, a AppStore, que disponibiliza aplicativos para iPhone e iPad, foram encontramos diferentes aplicativos associados ao termo BIM, dos quais foram selecionados três adequados ao objetivo da pesquisa, que são: BIM+ Explorer, BIM 360 GLUE e BIMx.

Nestes aplicativos foram então realizadas as comparações definidas pelos seguintes critérios: tipos de arquivo (A), usabilidade das ferramentas disponíveis (B) e possibilidades de visualização e interação com o modelo BIM (C).

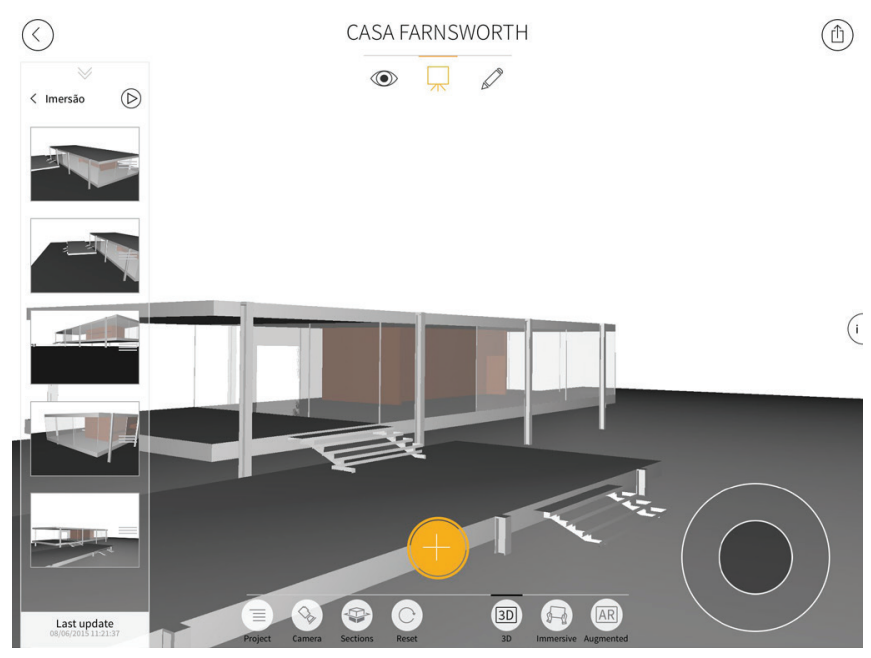

Figura 1: Visualização de modelo BIM com o aplicativo Bim + Explorer.

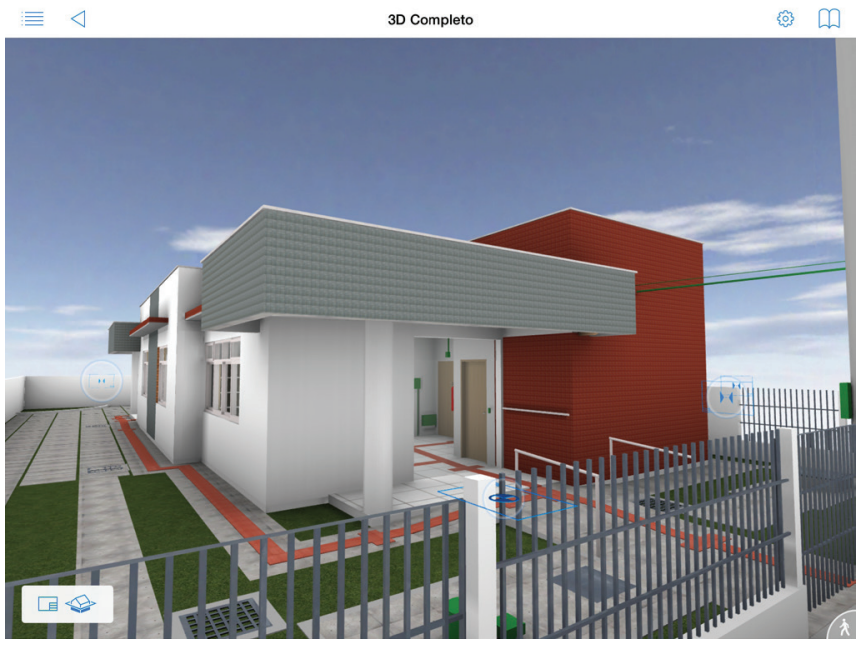

Figura 2: Visualização de modelo BIM com o aplicativo BIMx.
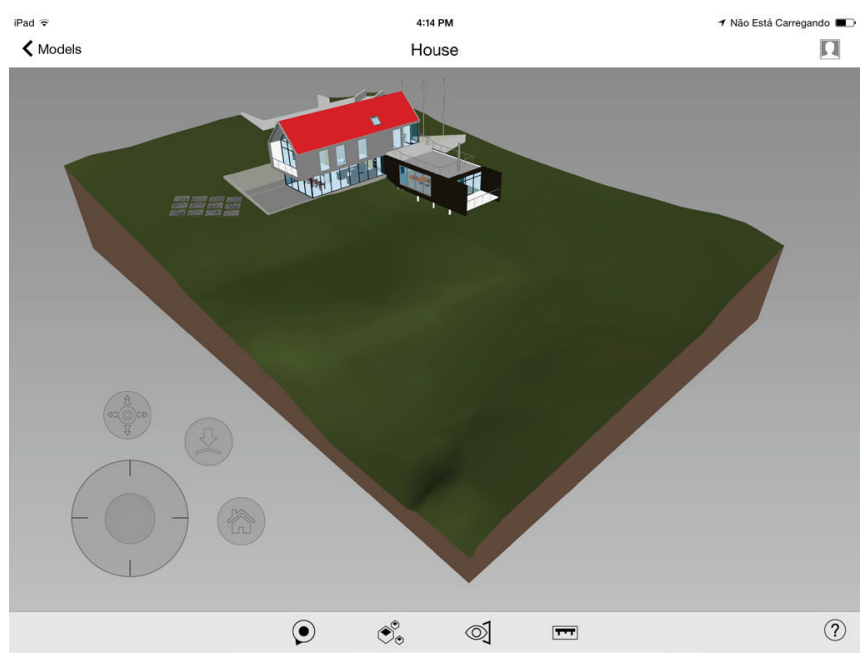

Figura 3: Visualização de modelo BIM com o aplicativo BIM 360 GLUE. 


\section{Resultados}

A análise inicial dos aplicativos selecionados para a realização do estudo demonstrou que estes possuem as seguintes características:

- BIM+Explorer:aplicativo desenvolvido pela Nemetschek, que possui 44,7 MB de quantidade de informação, sendo a versão mais atual a 2.4 .13 , com possibilidade de uso do aplicativo nos idiomas inglês e alemão;

- BIMx: aplicativo desenvolvido pela Graphisoft, que possui 23,2 MB de quantidade de informação, sendo versão mais atual a 3.6.2300, com possibilidade de uso do aplicativo nos idiomas inglês, espanhol e português entre outros;

- BIM 360 Glue: aplicativo desenvolvido pela Autodesk, que possui $63 \mathrm{MB}$ de quantidade de informação, sendo a versão mais atual a 3.1, com possibilidade de uso do aplicativo apenas no idioma inglês.

Todos os aplicativos testados possuem versões pagas e com recursos extras, os quais, em geral, são adequados para quando utilizados por equipe ou sistema multiusuário. No entanto, para esta pesquisa foi utilizada apenas a versão gratuita de cada aplicativo e os recursos próprios desta versão.

\section{Comparação relacionada ao critério ' $A$ '}

$\mathrm{Na}$ comparação dos tipos de arquivos possíveis de serem visualizados nos aplicativos, considerou-se mais relevantes, dentre os analisados, aqueles com extensões referentes a modelos BIM, ou seja, a extensão IFC (Industry Foundation Classes), já que este é o principal protocolo internacional utilizado nos dias de hoje para interoperabilidade de dados dos aplicativos e processos de projeto.

Mesmo que a cada versão do protocolo venha sendo observado progressões em termos de compatibilidade, tal protocolo ainda possui algumas restrições, como observa Kiviniemi et al. (2008), uma vez que determinadas informações de objetos geométricos ainda não são suportadas pelo formato, fazendo com que alguns destes modelos exibam elementos gráficos distintos quando comparados com o seu software de origem.

Neste sentido, verificou-se que no aplicativo BIM+ Explorer é possível realizar a visualização de modelos BIM desenvolvidos ou exportados na extensão *IFC; no aplicativo BIMx é possível realizar a visualização apenas do arquivo exportado pelo software de modelagem BIM - ArchiCAD que gera arquivo com extensão BIMX; e no que se refere ao aplicativo BIM 360 GLUE, uma considerável gama de extensões relacionados aos programas de computador desenvolvidas pela Autodesk são compatíveis, passíveis de visualização, mas os modelos BIM com possibilidade de serem visualizados são os desenvolvidos ou exportados na extensão ".IFC".

\section{Comparação relacionada ao critério ' $\mathrm{B}$ '}

No critério de comparação referente a usabilidade das ferramentas do aplicativo, o qual levou em consideração da dificuldade ou facilidade de manipulação das ferramentas presentes nos aplicativos selecionados.

Verificou-se que no aplicativo BIM+ Explorer as ferramentas de edição são disponibilizadas na parte superior da tela, logo abaixo do nome do projeto visualizado. Estas ferramentas possibilitam edições para organizar uma apresentação de determinada sequência de pontos de vista de câmeras organizados a partir do dispositivo, assim como fazer anotações sobre vistas salvas.

Nas laterais, o aplicativo apresenta mais opções de visualização e interação com o modelo tridimensional carregado. Na esquerda, vistas salvas no menu de edição podem ser arranjadas para apresentação do projeto. À direita, um pequeno ícone de ajuda que se sobrepõe à tela de projeto exibe a descrição de cada ícone, além de um duplo círculo grandequefunciona comouma manopla de controlemecânico, possibilitando 'caminhar' através do objeto arquitetônico e mudar o ponto de vista da câmera. Por fim, um outro pequeno ícone oferece a publicação ou compartilhamento da imagem que está sendo exibida, via e-mail ou redes sociais.

Na parte inferior da tela estão as principais ferramentas, que são: 'project', 'camera', 'sections', 'reset'. Estas ferramentas possibilitam respectivamente: gerenciamento do que é visto projeto por meio da modificação ocultar/exibir camadas; reposicionar vista conforme pontos de vistas de câmeras salvas previamente no programa de origem, selecionar cortes nos planos ' $\mathrm{X}$ ' ' $\mathrm{Y}$ ' e 'Z' e reposicioná-los conforme necessidade, e por fim um ícone '3D' que reestabelece o projeto ao seu estado de visualização inicial. Os ícones '3D', 'immersive' e 'augmented' oferecem opções de visualização do modelo tridimensional (conforme posicionamento da câmera inicial da abertura do arquivo), recurso de imersão e realidade aumentada.

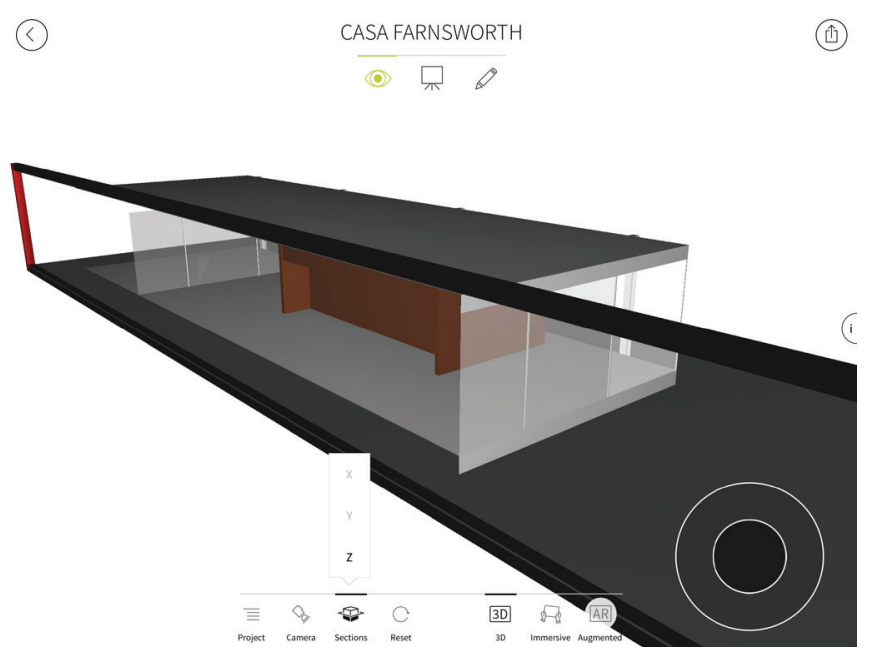

Figura 4: Tela do app Bim + Explorer Seção eixo 'Z'.

O aplicativo ainda oferece recursos extras de interação com o projeto, à medida que se mantém a tela pressionada sobre determinado ente geométrico do projeto. São exibidos, então, novo conjunto de ferramentas, dentre elas: ocultar/ exibir geometria selecionada, anexar arquivo de imagem, 
fazer comentários que são enviados ao servidor onde está hospedado o arquivo.

Quanto ao aplicativo BIMx, este disponibiliza, na versão analisada, ferramentas direcionadas à interação, com possibilidades de visualização do objeto arquitetônico. Possui duas barras de ferramentas: uma no topo, outra na base da tela. Na parte superior da tela, pode-se selecionar vistas do modelo utilizado, adicionar vistas de câmera (camera views) a um grupo de favoritas que possibilita retorno à visualização, salva quando necessário. O aplicativo permite, também, imprimir o que o que é exibido na tela e alterar o modelo de sombreamento entre 'realístico', 'opaco', 'simples' e metalizado.

$\mathrm{Na}$ barra inferior há ferramentas que possibilitam visualizar o projeto com um plano de corte horizontal ajustável na altura. $\mathrm{O}$ aplicativo permite selecionar objetos arquitetônicos, tais como janelas, portas, paredes, etc. disponibilizando informação extra (dimensão, área, classe, id a respeito do ente selecionado).

No aplicativo BIM 360 GLUE, as ferramentas disponibilizadas ficam na parte inferior da tela e são: gerenciamento de exibição do projeto conforme camadas ou disciplinas (arquitetônico, estrutural), gerenciamento de visualizações salvas, edição de revisão sobre elementos geométricos do projeto e medições de distâncias ou áreas, ferramentas que possibilitam, por exemplo, conferir se há algum conflito interdisciplinar que pode ser relatado e compartilhado a partir do aplicativo. É possível, ainda, obter informações complementares, tais como: dimensões, material, espessura, etc., a partir da seleção de um ente geométrico do modelo tridimensional.

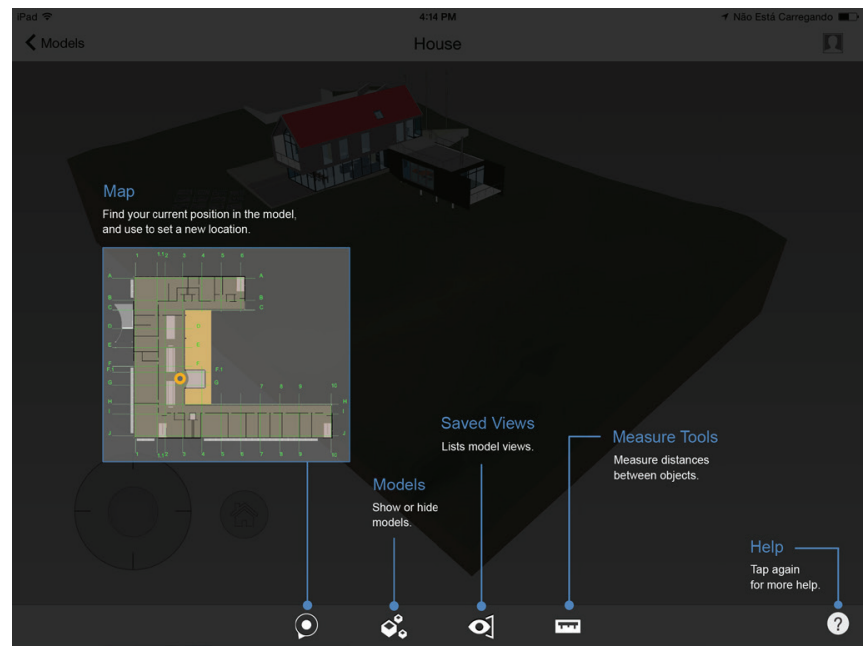

Figura 5: Tela do app BIM 360 Glue - Tela de ajuda.

\section{Comparação relacionada ao critério ' $\mathrm{C}$ '}

Os aplicativos investigados oferecem semelhante maneira de interagir com o projeto visualizado no que diz respeito ao toque na tela do dispositivo. Aproximar/afastar, deslocar e rotacionar são disponibilizados da mesma maneira em todos.
Na verificação quanto às possibilidades de visualização e interação com o modelo BIM em cada aplicativo, se constatou que no caso do aplicativo BIM+ Explorer há a possibilidade de interação com planos de corte nos eixos X, Y e Z, recursos de visualização imersiva e realidade aumentada que funcionam seguindo a instrução para criação de uma etiqueta para vincular a um ponto específico do projeto.

O aplicativo permite ainda que sejam feitas anotações com canetas coloridas virtuais que ficam anexadas junto ao arquivo, no projeto que está hospedado num servidor na internet. Portanto, as referidas anotações ficam visíveis a qualquer componente da equipe de trabalho, assim como anexos que podem ser vinculados a entidades geométricas visíveis no modelo tridimensional. Ademais, é possível salvar vistas de câmeras a qualquer instante durante a interação com o modelo 3D dentro do aplicativo.

OappBIMxdemonstrater seufocovoltadoaumainteração do modelo tridimensional construído no $\operatorname{ArchiCAD} \AA$, que possibilita caminhar pelo projeto por intermédio do botão que simula a utilização de uma manopla posicionada na parte inferior da tela, além de obter informações de elementos geométricos tipificados como elementos construtivos (paredes, janelas, portas, pisos, etc.), assim como gerar um corte no plano horizontal e posicioná-lo em diferentes alturas.

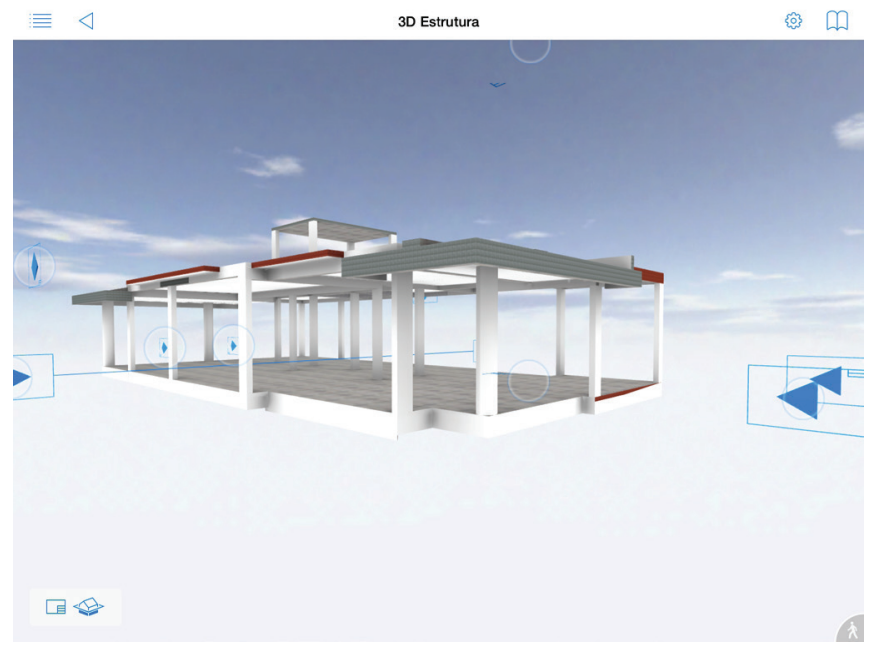

Figura 6: Tela do aplicativo BIMx - Vista salva previamente no software de origem.

No aplicativo BIM 360 GLUE, há a possibilidade de interagir com o modelo tridimensional através da seleção de disciplinas (arquitetura, estrutura), conforme qualidade das informações que o modelo construído em software BIM possuir. Adição de comentários textuais ou seleções gráficas também são permitidas, sendo salvas e compartilhadas com membros da equipe de projeto instantaneamente. Entrega ferramentas de conferência ou comparação com o objeto arquitetônico construído, tais como: trena digital e imersão in loco, por meio de posicionamento de georreferência de projeto. 


\section{Considerações finais}

Os aplicativos investigados são bastante semelhantes no que se refere às possibilidades de visualização e interação com um modelo BIM carregado e manipulado nesta investigação. Não obstante, diferem em alguns aspectos importantes, conforme os critérios de comparação adotados, o que faz com que cada um, em função destas distinções, tenha seu foco funcional direcionado em razão do seu modus operandi.

Nestas verificações, fora constatado que o aplicativo $\mathrm{BIM}+$ Explorer oferece condições gerais de usabilidade e ferramentas abrangentes para visualização e interação aluno/ professor num atelier de uma disciplina de projeto.

Entretanto, os aplicativos BIMx e BIM 360 GLUE oferecem recursos com potenciais distintos de visualização e interação. O BIMx mostra-se mais eficiente no que tange à apresentação do projeto, por oferecer uma visualização diferenciada quanto à qualidade gráfica do ambiente e do modelo BIM, além de poder ser visualizado em smartphones com sistema Android ou iOS, o que aumenta sua abrangência de uso e o direciona para um uso arquiteto/clientes.

O BIM 360 GLUE tem seu potencial mais relacionado com as ferramentas que oferece, devido a estas possibilitarem uma interação qualificada no que diz respeito a detalhes técnicos do projeto representado no modelo tridimensional. Além disto, possibilita integração com variada extensão de arquivos de outros programas de computador (mesmo que fora do paradigma BIM), além do compartilhamento de informações diretamente do aplicativo que podem ser acessadas por todos os membros de uma equipe, o que o direciona para um uso entre o arquiteto e os demais envolvidos na construção efetiva do projeto.

\section{Referências}

Alexie, S. (1992). The business of fancydancing: Stories and poems. Brooklyn, NY: Hang Loose Press.

AllPlan BIM+Explorer. BIM+Explorer Acessado em 20 jun. 2015. Online. Disponível em: https://www.bimplus.net//out/ pictures/guide/guide_en/01.bimplus_starter_guide.pdf

Autodesk 360 GLUE. 360 GLUE. Acessado em 20 jun. 2015. Disponível em: http://www.autodesk.com/products/bim360-glue

Cattani, A. (2001). Recursos informáticos e telemáticos como suporte para formação e qualificação de trabalhadores da construção civil. Tese (Doutorado em Informática na Educação) - Porto Alegre: UFRGS, CINTED, PGIE.

Cattani, A; Antoniazzi, A; Pedone, J.V.C.; Costa, A. E.; Hayet, P.V. (2007). Comparação entre ferramentas de modelagem computacional em pesquisa sobre ambientes históricos. SIGraDi - Proceedings of the 11th Iberoamerican Congress of Digital Graphics, México.

Filho, C.A. (2009) Acesso ao modelo integrado do edifício. Dissertação de mestrado. Pós-Graduação em Construção Civil - Setor de Tecnologia, Universidade Federal do Paraná.

Florio, W. (2007). Contribuições do Building Information Modeling no processo de Projeto em Arquitetura. In: Encontro de Tecnologia de Informação e Comunicação na Construção Civil. Porto Alegre. CD-Rom, Anais, Rio Grande do Sul.

Graphisoft BIMx. BIMX_PRO FLYER. Acessado em 20 jun. 2015. Disponível em: http://www.graphisoft.com/ftp/ marketing/bimx/BIMx_PRO_Flyer.pdf

Kiviniemi, A. (2007) Support for Building Elements in the IFC $2 \times 3$. Implementations based on 6th Certification Workshop Result in May 2007. VTT, 2007. Disponível em: http://www. coinsweb.nl/downloads/IFC_2x3_Data_Exchange.doc Lévy, P. (1996). O que é o virtual? Titulo original: Qu'est-ce que Le virtuel? Tradução: Paulo Neves. São Paulo. Editora 34 Lévy, P. (1999). Cibercultura. Titulo original: Cyberculture. Tradução: Carlos Irineu da Costa. Rio de Janeiro: Editora 34 Souza, C. (2005). The Semiotic Engineering of HumanComputer Interaction (Acting with Technology), The MIT Press

Veliz, A., Kocaturk, T., Medjdoub, B. e Balbo, R. (2012). Dialogs between Physical and Digital Modelling Methods on Architectural Design. Proceedings of the 30th eCAADe Conference, Vol. 2, 281-289. 